\title{
In vitro fertilization and embryo culture in the grey short-tailed opossum, Monodelphis domestica
}

\author{
H. D. M. Moore ${ }^{*}$ and D. A. Taggart $\dagger$ \\ Institute of Zoology, Regent's Park, London NW1 4RY, UK
}

\begin{abstract}
In vitro fertilization and early embryo culture was undertaken in the South American marsupial, the grey short-tailed opossum, Monodelphis domestica. Adult females were induced into oestrus by a system of pairing with an unfamiliar male and mature oocytes were recovered from the ovary $15-18 \mathrm{~h}$ after mating and placed in pre-warmed modified MEM medium at $33^{\circ} \mathrm{C}$ (basal body temperature) or $37^{\circ} \mathrm{C}$. Spermatozoa recovered from the cauda epididymides of adult males were preincubated in medium for $2 \mathrm{~h}$ during which time paired spermatozoa separated and initiated hyperactivated motility. Oocytes were transferred to $0.4 \mathrm{ml}$ drops of spermatozoa containing $0.5-1.0 \times 10^{6}$ spermatozoa $\mathrm{ml}^{-1}$. Only single spermatozoa bound to the zona pellucida, and fertilization occurred within $1-2 \mathrm{~h}$ as indicated by a breach in the zona and confirmed by electron microscopy. At $37^{\circ} \mathrm{C}, 95$ of $152(62.5 \%)$ oocytes were fertilized and $64(67 \%)$ developed to two-cell stage or beyond. At $33^{\circ} \mathrm{C}, 5$ of $28(18 \%)$ oocytes were fertilized. This is the first report of complete in vitro fertilization in a marsupial.
\end{abstract}

Introduction

Since the first report of complete in vitro fertilization in hamsters (Yanagimachi and Chang, 1964), the technique has been applied successfully to an increasing number of mammalian species. However, nearly all in vitro studies have been performed with gametes from placental (eutherian) species, with very few investigations carried out in the remaining mammals, the marsupials (Metatheria) and monotremes (Prototheria). These last two groups represent about $6 \%$ of extant mammalian species and have evolved reproductive processes distinct from those of Eutheria (see Tyndale-Biscoe and Renfree, 1987; Rodger, 1991). In a previous study, spermatozoa recovered from the oviduct of the Virginian opossum, Didelphis virginiana, were shown to be capable of penetrating the zona pellucida of pre-ovulatory oocytes in vitro, but fertilization using epididymal or ejaculated spermatozoa was not attempted (Rodger and Bedford, 1982). Here, we report, for the first time, complete in vitro fertilization and the subsequent culture of zygotes in a marsupial, the grey short-tailed opossum, Monodelphis domestica. This small (90-150 g) marsupial is a member of the family Didelphidae, and in common with all but one of the American marsupials has spermatozoa that form pairs during epididymal maturation (Temple-Smith and Bedford, 1980; Taggart, 1991; Moore, 1992). Because the grey short-tailed opossum can be maintained under laboratory conditions and is easy to handle, it has rapidly become established as the animal of choice for the study of New World marsupial biology (Moore, 1992).

${ }^{*}$ Present address: Department of Molecular Biology and Biotechnology, University of Sheffield, PO Box 594, Western Bank, Sheffield S10 2UH, UK.

†Present address: Department of Anatomy and Histology, University of Adelaide, Adelaide, South Australia.

Received 1 September 1992.

\section{Materials and Methods}

\section{Animals}

All the animals used in this study were from a laboratory colony of grey short-tailed opossums, Monodelphis domestica, established in 1983 at the Institute of Zoology. Housing, lighting and dietary requirements are described in detail elsewhere (Baggott et al., 1987; Moore, 1992). Briefly, adult opossums (males: > $120 \mathrm{~g} / 7$ months; females: $>60 \mathrm{~g} / 5$ months) were kept singly in rat cages $(45 \times 25 \mathrm{~cm})$ at $25-28^{\circ} \mathrm{C}$ in $80 \%$ relative humidity and under artificial light $(12 \mathrm{~h}$ light: $12 \mathrm{~h}$ dark with light from 19:00 to 07:00 h). Diet consisted of a fox food pellet (Kemovit, Bristol) given ad libitum and fresh minced beef given once a week.

\section{Induction of oestrus}

Oestrus was induced in adult females by a system of pairing with an unfamiliar male (Baggott et al., 1987) and mating was monitored by time-lapse video recording (Panasonic ET 180). Ovulation occurred 18-24 h after the first mating (Baggott and Moore, 1990).

\section{In vitro fertilization (IVF)}

The IVF medium consisted of modified MEM medium (Gibco, Life Technologies Ltd, Paisley) consisting of $105.6 \mathrm{mmol} \mathrm{NaCl} \mathrm{l}^{-1}\left(6.17 \mathrm{~g} \mathrm{l}^{-1}\right), 5.06 \mathrm{mmol} \mathrm{KCl} \mathrm{l}{ }^{-1}(0.378 \mathrm{~g}$ $\left.\mathrm{l}^{-1}\right), 0.73 \mathrm{mmol} \mathrm{CaCl} \mathrm{l}^{-1}\left(0.226 \mathrm{~g} \mathrm{l}^{-1}\right), 1.17 \mathrm{mmol} \mathrm{KH}_{2} \mathrm{PO}_{4} \mathrm{l}^{-1}$ $\left(0.16 \mathrm{~g} \mathrm{l}^{-1}\right), \quad 1.01 \mathrm{mmol} \mathrm{MgSO}_{4} \cdot 7 \mathrm{H}_{2} \mathrm{O} \quad \mathrm{I}^{-1}\left(0.25 \mathrm{~g} \mathrm{l}^{-1}\right)$, $25.3 \mathrm{mmol} \mathrm{NaHCO} \mathrm{l}^{-1}\left(2.15 \mathrm{~g} \mathrm{l}^{-1}\right), 0.27 \mathrm{mmol} \mathrm{Na}$ pyruvate $1^{-1}\left(0.03 \mathrm{~g} \mathrm{l}^{-1}\right), 21.6 \mathrm{mmol} \mathrm{Na}$ lactate $1^{-1}\left(2.42 \mathrm{~g} \mathrm{l}^{-1}\right)$, 
$5.56 \mathrm{mmol}$ glucose $\mathrm{l}^{-1}\left(1.0 \mathrm{~g} \mathrm{l}^{-1}\right)$ and $4.0 \mathrm{~g} \mathrm{BSA}^{-1}$. Penicillin (1000 iu ml ${ }^{-1}$ ) and Streptomycin $\left(50 \mu \mathrm{g} \mathrm{ml}^{-1}\right.$ ) were added before filtration through a $0.22 \mu \mathrm{m}$ filter. The medium had a $\mathrm{pH}$ of 7.4, and an osmolarity of 330 mosm $\mathrm{l}^{-1}$ when gassed with $5 \% \mathrm{CO}_{2}$ and air, and prewarmed to a temperature of $33^{\circ} \mathrm{C}$ (opossum basal body temperature) or $37^{\circ} \mathrm{C}$.

Fifteen to eighteen hours after mating, a female opossum was killed by injection with Euthatal (May and Baker, Dagenham) and its reproductive tracts removed and placed in IVF medium prewarmed to $33^{\circ} \mathrm{C}$ or $37^{\circ} \mathrm{C}$ and gassed with $5 \% \mathrm{CO}_{2}$. The ovaries were dissected free and placed in fresh medium, and under a dissecting microscope the largest follicles were carefully punctured with a fine glass pipette. Naked oocytes devoid of cumulus cells were immediately transferred to a $200 \mu$ ldrop of fresh medium under silicone oil (Dow Corning) and placed in an incubator in $5 \% \mathrm{CO}_{2}$ in air. A sexually mature, adult male opossum was killed as described above. The epididymides were removed and washed in IVF medium to remove any traces of blood. The cauda epididymides were then divided into four equal segments and each placed in a $200 \mu \mathrm{l}$ drop of medium under silicone oil. Several incisions were gently made into each tissue segment to assist in the release of spermatozoa from the duct. After incubation for $1 \mathrm{~min}$ in $5 \% \mathrm{CO}_{2}$ and air at $33^{\circ} \mathrm{C}$ or $37^{\circ} \mathrm{C}$, each epididymal segment was removed, placed in a second drop of medium and returned to the incubator. Five minutes later the epididymal tissue was removed and discarded and each drop of spermatozoa incubated for a further $2 \mathrm{~h}$ in $5 \%$ $\mathrm{CO}_{2}$ in air. At the end of this period, medium was carefully replaced with fresh IVF medium. This was done with a minimal loss of spermatozoa as most (paired and single) had attached loosely by the head to the bottom of the culture plate and the spermatozoa resuspended in the drop by gentle pipetting. The sperm concentration in those drops containing strongly motile spermatozoa was determined using an Improved Neubauer counting chamber.

Oocytes (2-5) were transferred into the appropriate drops $(0.4 \mathrm{ml})$ of spermatozoa containing $0.5-1.0 \times 10^{6}$ spermatozoa $\mathrm{ml}^{-1}$ and monitored every $30 \mathrm{~min}$ for evidence of fertilization. At the same time observations were made of the proportion of single or paired spermatozoa in each drop and of sperm motility. After incubation for $4 \mathrm{~h}$, oocytes were transferred to fresh medium under oil.

\section{Light and electron microscopy}

Sperm-egg interactions were examined and photographed by phase contrast microscopy. Eggs penetrated by spermatozoa were transferred to fresh medium for further incubation or were fixed in $2 \%$ glutaraldehyde in $0.05 \mathrm{~mol}$ phosphate buffer $\mathrm{l}^{-1}$ (pH 7.4, 330 mosm $~^{-1}$ adjusted by addition of sucrose) overnight at $4^{\circ} \mathrm{C}$, followed by secondary fixation in $1 \%$ osmium tetroxide for $4 \mathrm{~h}$ at $4^{\circ} \mathrm{C}$, rapid dehydration through a series of alcohols (as for routine EM) and embedding in Epon-Araldite (Mollenhauer, 1964).

Selected eggs were serially sectioned $(1 \mu \mathrm{m})$, stained in toluidine blue and examined with an Olympus BH-2 light microscope. When sections of interest were found, a corresponding series of ultra-thin sections $(65-70 \mathrm{~nm})$ were cut and stained in aqueous uranyl acetate $(12 \mathrm{~min})$ and lead citrate $(8 \mathrm{~min})$ and examined in a Jeol JEM-1200 EX electron microscope.

\section{Results}

\section{Motility and unpairing of spermatozoa in vitro}

The proportion of spermatozoa recovered from the distal cauda epididymidis and vas deferens displaying progressive motility in IVF medium was $82 \pm 8 \%$ (from eight males). Most spermatozoa were in pairs, $78 \pm 9 \%$, which exhibited rapid forward motility ( $>300 \mu \mathrm{s}^{-1}$ ) with synchronized flagella beat (D. A. Taggart and H. D. M. Moore, unpublished) when not attached to the bottom of the culture dish. After incubation in IVF medium for $2 \mathrm{~h}$ at $37^{\circ} \mathrm{C}$, the proportion of paired spermatozoa fell significantly $(P<0.05)$ to $63 \pm 12 \%$, while there was a corresponding increase in motile but single spermatozoa (Fig. 1). During this stage, some paired spermatozoa (about 15\%) exhibited erratic, nonprogressive motility with asynchronous tail beats and their heads showed signs of imminent uncoupling. After incubation for $4 \mathrm{~h}$, the proportion of paired spermatozoa had decreased further (compared with 0 and $2 \mathrm{~h}$ ) to $46 \pm 7 \%$ $(P<0.005)$. Single spermatozoa at this time were usually progressively motile but also displayed periods of erratic 'whiplash' tail movement with nonprogressive motility. After $24 \mathrm{~h}$ the proportion of paired spermatozoa decreased to $22 \pm 8 \%$. These spermatozoa displayed active tail beating but were invariably attached to the bottom of the culture dish. Single spermatozoa were immotile at this stage.

Spermatozoa incubated at $33^{\circ} \mathrm{C}$ initially displayed a similar forward progressive motility $\left(>250 \mu \mathrm{m} \mathrm{s}^{-1}\right)$ but the proportion of spermatozoa exhibiting erratic motility after 2 or $4 \mathrm{~h}$ incubation remained low $(<5 \%)$ and few spermatozoa were unpaired $(<10 \%)$. After $24 \mathrm{~h}$ in culture, the proportion of motile paired spermatozoa was $32 \pm 12 \%$.

\section{Recovery of oocytes}

From 19 females, 162 pre-ovulatory oocytes were recovered, giving an average of 4.76 oocytes per ovary. Most oocytes on release from the follicle were devoid of follicular cells and exhibited a first polar body (88\%), indicating that egg maturation was nearing completion. Oocytes obtained within $15 \mathrm{~h}$ after mating were invariably surrounded by a corona of follicular cells and were at the germinal vesicle stage. When these oocytes were denuded of follicular cells by mechanical removal (repeated aspiration of oocytes in a narrow bore pipette), spermatozoa failed to bind to their surface (20 oocytes examined; H. D. M. Moore, unpublished observation). Mature intact oocytes were $230 \pm 12 \mu \mathrm{m}$ in diameter with a peripheral ooplasm containing many mitochondria and an inner yolky region full of dense lipid bodies.

\section{Sperm-egg binding, fertilization and embryo culture}

Fully mature oocytes (without follicular cells) were transferred to drops of spermatozoa previously incubated in IVF medium for $2 \mathrm{~h}$. Motile single spermatozoa immediately bound to the zona surface by their flat acrosomal face (Taggart et al, in press). Paired spermatozoa displayed progressive motility, but failed to attach to oocytes. Sperm penetration of oocytes was initiated over $2-3 \mathrm{~h}$, depending on the preparation, and was detected by the presence 


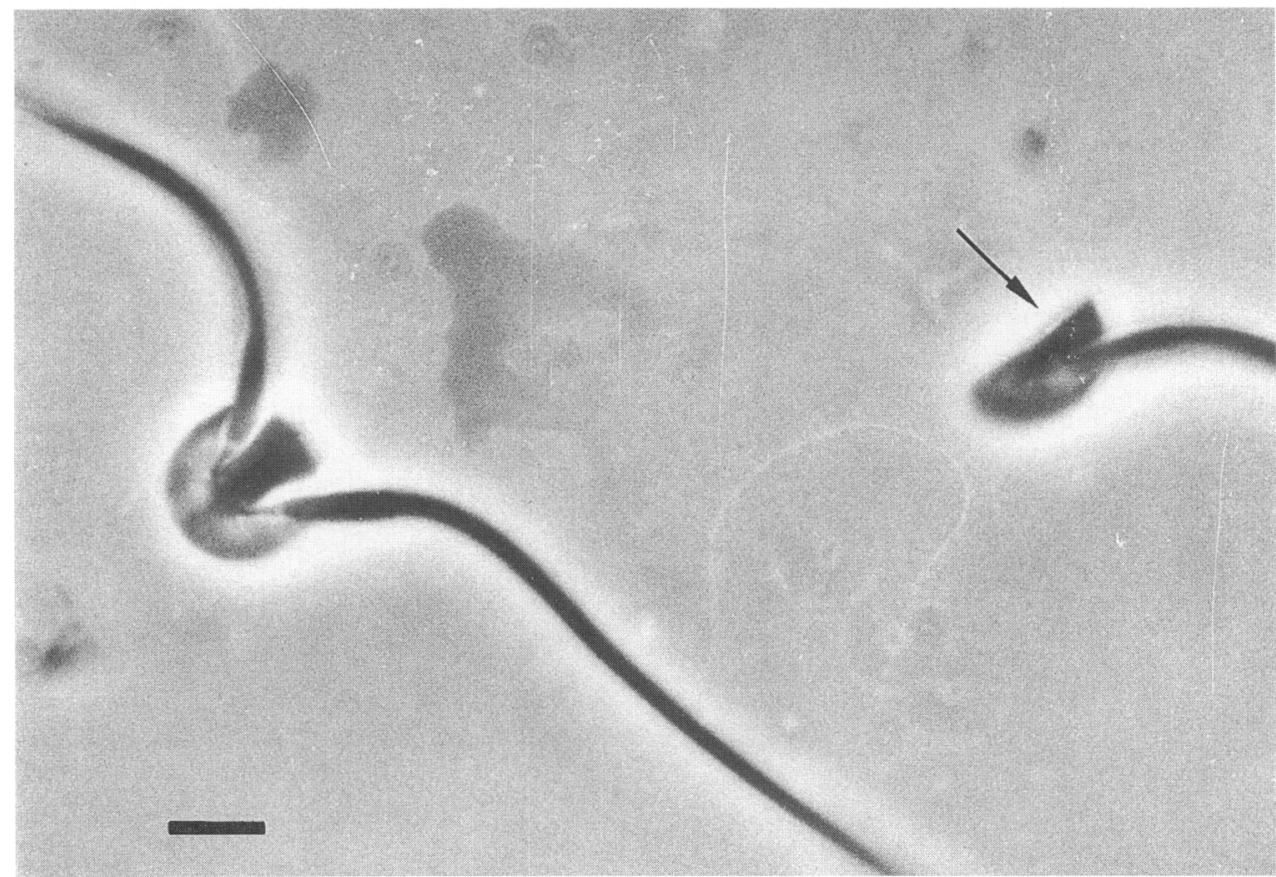

Fig. 1. Phase contrast micrograph of paired and single spermatozoa of grey short-tailed opossum. Single spermatozoa bound to the zona surface of mature oocytes with their flat acrosomal surface (acrosome arrowed). Bar $=1 \mu \mathrm{m}$.

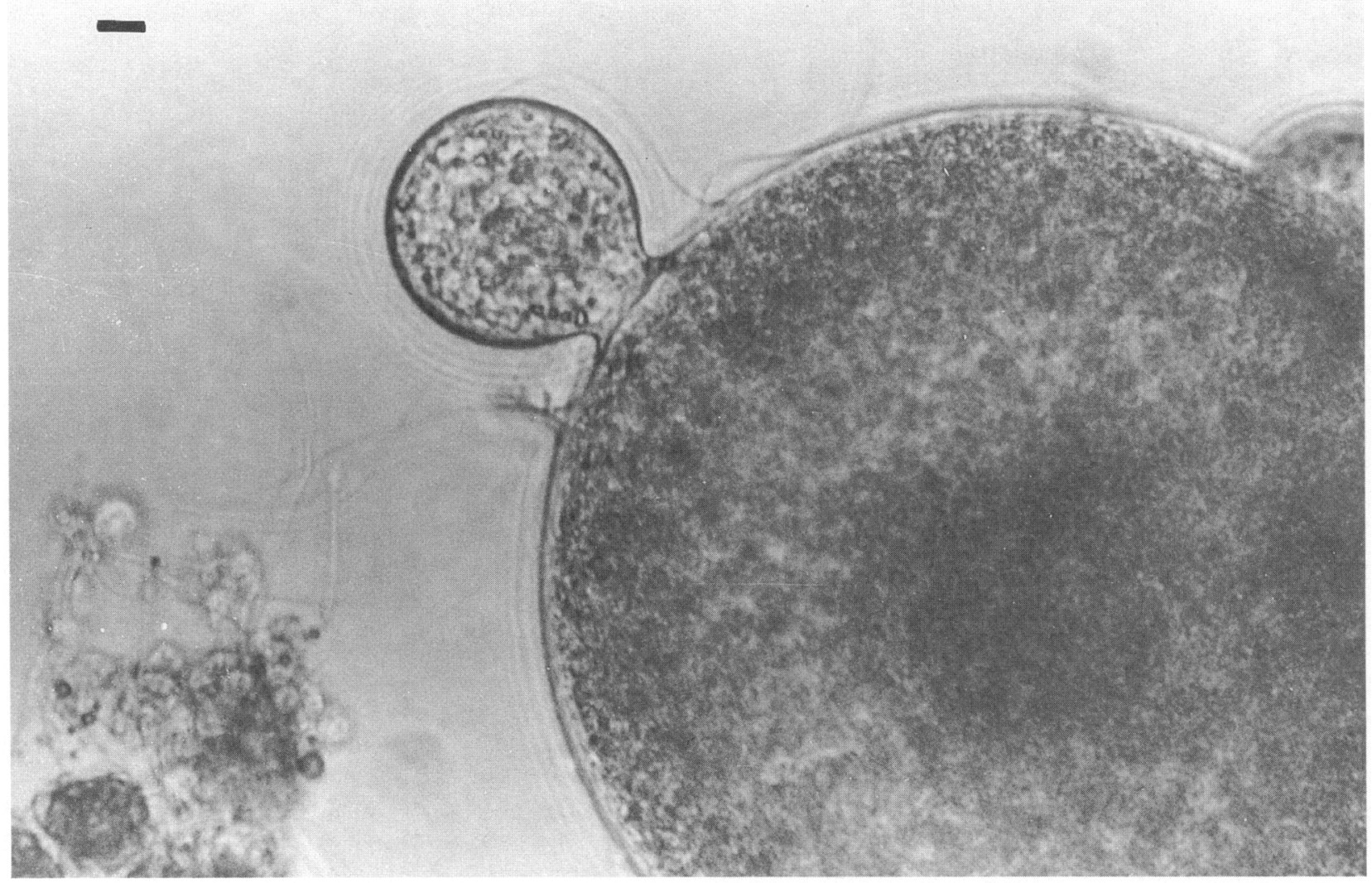

Fig. 2. Phase contrast micrograph of an oocyte of the grey short-tailed opossum immediately after fertilization. Ooplasm is extruding from the site of penetration. After about an hour this extrusion diminishes (see Fig. 3). Bar $=10 \mu \mathrm{m}$. 


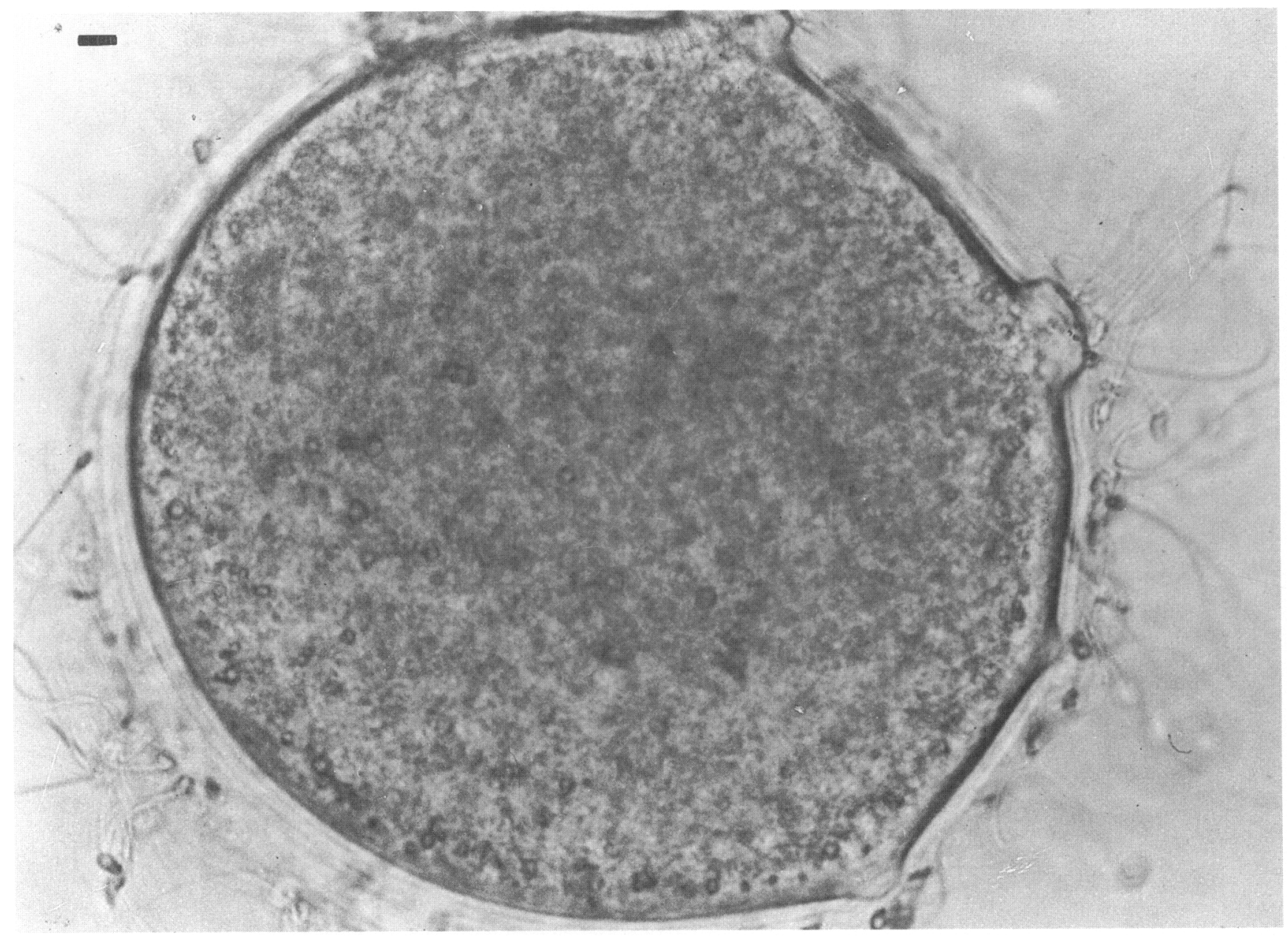

Fig. 3. A light micrograph of an oocyte of the grey short-tailed opossum penetrated by spermatozoa. Note the multiple breaches in the zona with the associated extrusion of ooplasm. Bar $=10 \mu \mathrm{m}$.

of a breach in the zona pellucida with the extrusion of cytoplasm (Fig. 2). Multiple penetration sites were apparent for many eggs incubated with spermatozoa for more than $2 \mathrm{~h}$ (Fig. 3). In eighteen incubations at $37^{\circ} \mathrm{C}, 95$ of $152(62.5 \%)$ oocytes showed clear signs of sperm penetration with a breached zona pellucida and the presence of a sperm tail in the vitellus. Sperm fusion with the oolemma was confirmed in a small proportion of these eggs (seven) by electron microscopy (Figs 4 and 5). Of the 95 oocytes fertilized, $64(67 \%)$ underwent activation with extrusion of a second polar body, yolky cytoplasmic breakdown (deutoplasmolysis) and subsequent cell division to two cells or beyond (Table 1). Because of the dense lipid content of the egg, pronuclei could not be readily visualized, although under phase contrast microscopy the movement of peripheral ooplasm to one pole was evident (Baggott and Moore, 1990). Oocytes judged unfertilized failed to activate even after several days of culture. The rate of development (Table 2) was similar to that of in vivo fertilized oocytes (Baggott and Moore, 1990) but embryos arrested at the 16-32 cell stage as unilaminar blastocysts were about to form (Fig. 6). Mature oocytes incubated in the absence of spermatozoa did not show perforation of their zona pellucida, and failed to undergo cleavage even after prolonged culture ( 7 days).

In three experiments with oocytes incubated with spermatozoa at $33^{\circ} \mathrm{C}, 5$ of $28(18 \%)$ oocytes were clearly fertilized. As this rate of fertilization was considerably lower than at $37^{\circ} \mathrm{C}$, most experiments were carried out at the higher temperature.

\section{Discussion}

As in nearly all American marsupials, the spermatozoa of the grey short-tailed opossum form into pairs during epididymal maturation such that their flat acrosomal surfaces are in close apposition (D. A. Taggart and H. D. M. Moore, unpublished). On release from the vas deferens and cauda epididymidis, these conjugated spermatozoa exhibit rapid progressive motility $\left(300 \mu \mathrm{m} \mathrm{s}^{-1}\right)$ with coordinated tail beats, which for a small proportion of cells was sustained for up to $24 \mathrm{~h}$ in fertilization medium in vitro. Generally, however, most sperm pairs began to display a characteristic erratic motility pattern after incubation for 2-4 h, closely reminiscent of hyperactivated motility observed for spermatozoa of eutherian species (see Yanagimachi, 1988). This alteration in sperm motility was normally associated with the uncoupling of the spermatozoa, but it was unclear whether sperm separation was precipitated by the change in flagella movement, or conversely, that a failure in cell-cell communication as the sperm heads dissociated prompted erratic uncoordinated motility. Sperm motility changes and unpairing were 


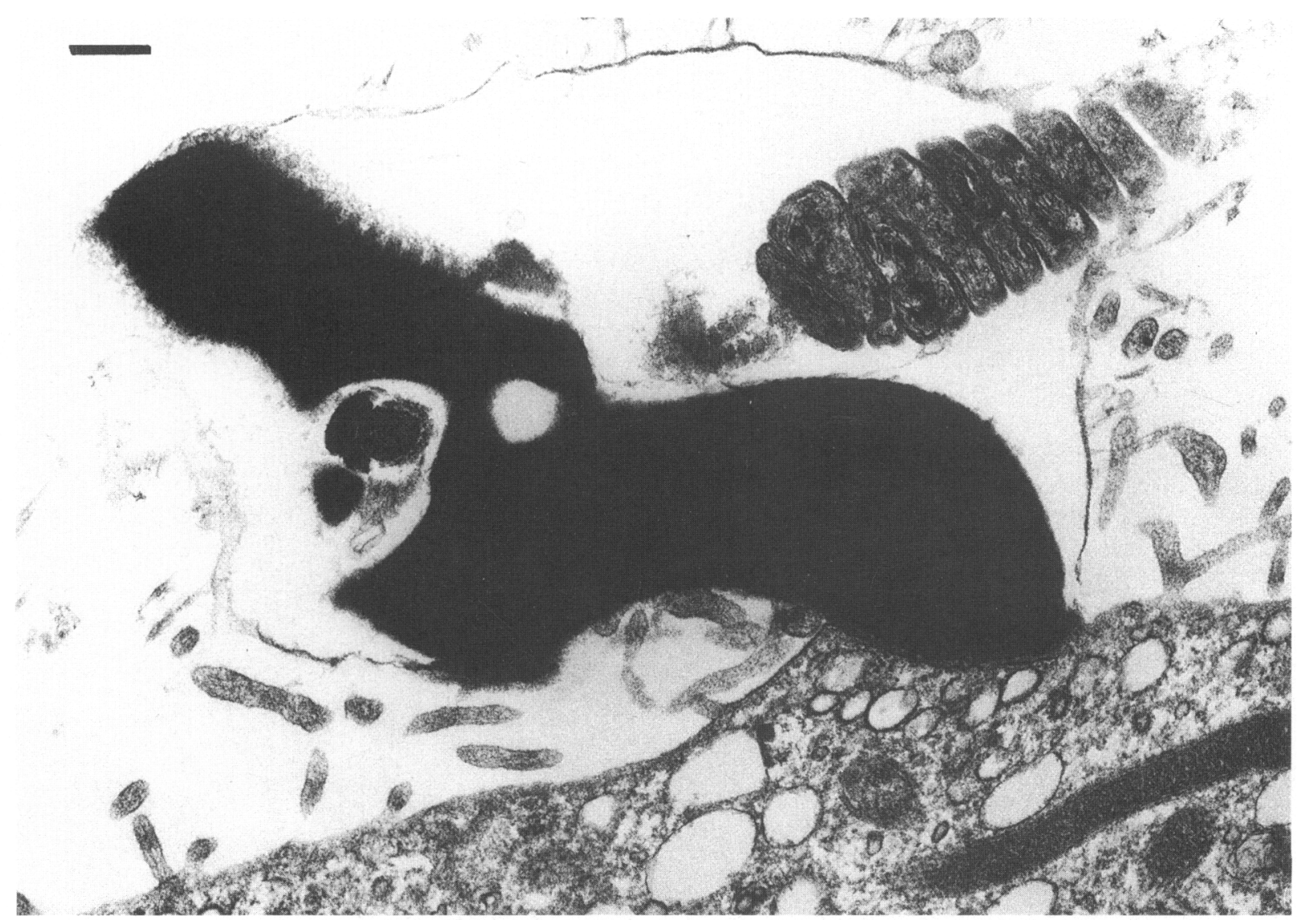

Fig. 4. An electron micrograph of initial sperm-egg fusion of the grey short-tailed opossum. Bar $=500 \mathrm{~nm}$.

initiated to a much greater extent at $37^{\circ} \mathrm{C}$ than at $33^{\circ} \mathrm{C}$ even though the latter temperature represented the basal body temperature of Monodelphis. The reason for this is unclear. At $37^{\circ} \mathrm{C}$ the survival of spermatozoa was not significantly less than at $33^{\circ} \mathrm{C}$. One possibility is that the higher temperature may therefore have accelerated biochemical changes (capacitation?) to spermatozoa without compromising their viability. Fertilization studies carried out in Didelphis (Rodger and Bedford, 1982) were also undertaken at $37^{\circ} \mathrm{C}$.

Single spermatozoa maintained a hyperactivated motility but had a shorter survival time in fertilization medium in vitro. Moreover, only after pair separation were spermatozoa capable of binding to the zona pellucida and subsequently fertilizing a mature oocyte. This sequence of events suggests that the process of sperm uncoupling might be analogous to capacitation for eutherian spermatozoa (see Yanagimachi, 1988). Certainly, the acrosomes of conjugated spermatozoa, isolated from their immediate surroundings by the desmosome-like union at the periphery of each sperm head (Temple-Smith and Bedford, 1980; Taggart, 1991) are protected from the microenvironment. Thus, in the same way that capacitation (in Eutheria) ensures that previously stable sperm membranes become sufficiently labile to undergo the acrosome reaction, unpairing exposes opossum sperm acrosomal membranes making them vulnerable perhaps to extrinsic factors. Unfortunately, under the phasecontrast microscope, the acrosomal status of a motile opossum spermatozoon was difficult to assess owing to the morphology of the sperm head. However, electron microscopy indicated that most single spermatozoa remain intact and exhibit no acrosomal breakdown in vitro unless they encounter an oocyte (Taggart et al., in press). In Australian marsupials, spermatozoa do not conjugate but they are also resistant to undergoing an acrosome reaction in a variety of capacitating media despite some vesiculation of acrosomal contents (Mate and Rodger, 1991). It therefore remains unclear whether sperm pairing has primarily evolved to protect the acrosome and to act as a capacitating mechanism or for some other reason. Recent evidence might support an alternative argument that pairing confers a distinct advantage on spermatozoa when swimming through the viscous fluids they might encounter in the female tract (D. A. Taggart and H. D. M. Moore, unpublished).

Single spermatozoa bound firmly to the zona pellucida of mature oocytes immediately their flat acrosomal surface made contact with the zona surface. It would seem that the acrosome reaction was initiated at this stage as sperm penetration of the zona matrix occurred rapidly, readily identified by the extrusion of ooplasm at the site of sperm entry. Electron microscopy has since confirmed these observations (Taggart et al., in press). Certainly, a zona-induced acrosome reaction would be an important requirement in the opossum. The oocyte is naked at ovulation (Baggott et al., 1987; Baggott and Moore, 1990), having no cumulus mass surrounding it that might otherwise prepare the membranes of fertilizing spermatozoa (see Yanagimachi, 1988). Perhaps more significantly, a mucoid 


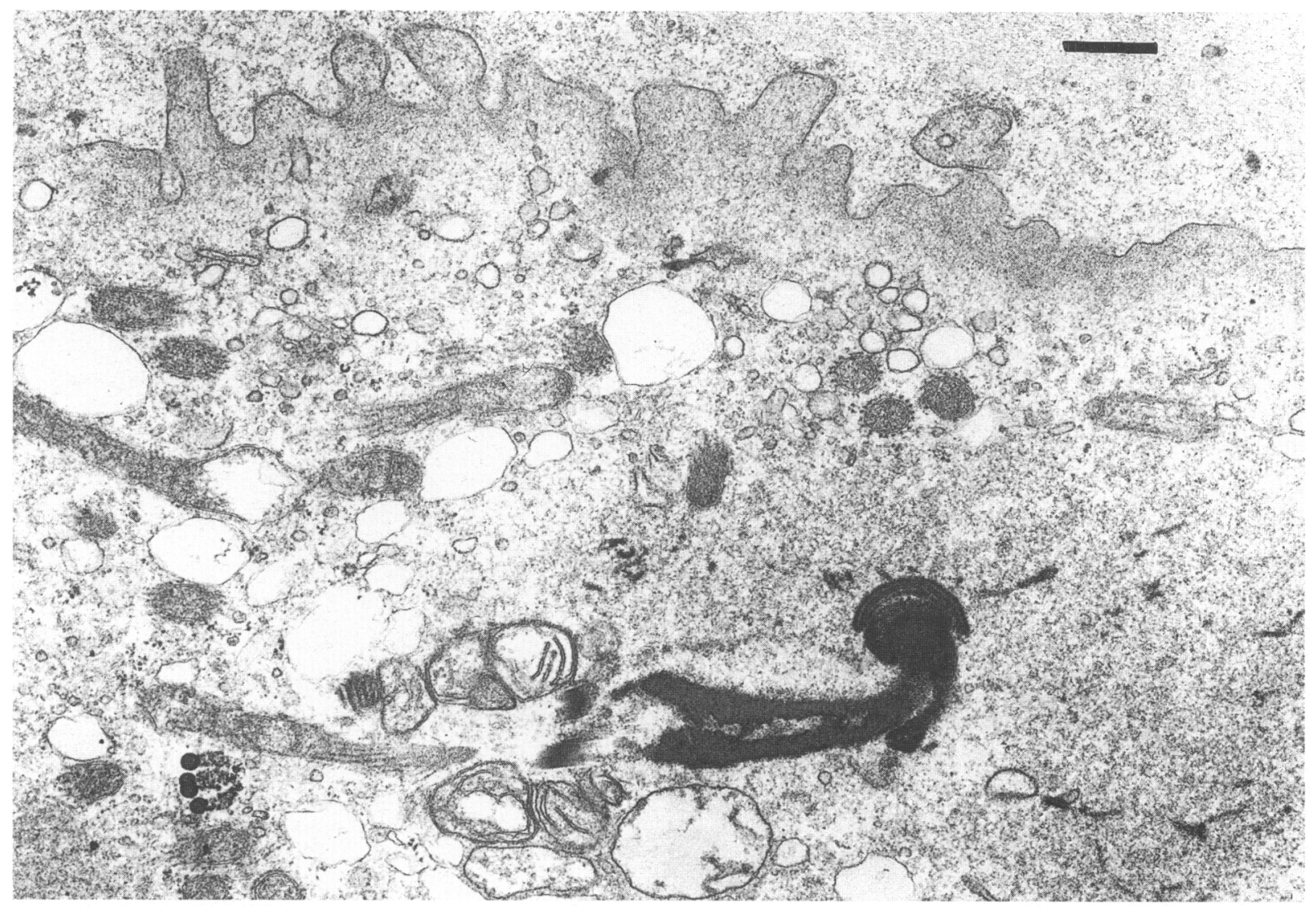

Fig. 5. An electron micrograph of a decondensing sperm head in the ooplasm of an oocyte of the grey short-tailed opossum, $2 \mathrm{~h}$ after insemination. Bar $=500 \mathrm{~nm}$.

Table 1. In vitro fertilization and embryo culture in the grey short-tailed opossum, Monodelphis domestica

\begin{tabular}{lccc}
\hline $\begin{array}{l}\text { Number of } \\
\text { experiments } \\
\text { (temperature) }\end{array}$ & $\begin{array}{c}\text { Oocytes } \\
\text { fertilized } \\
(\%)\end{array}$ & $\begin{array}{c}\text { 2-cell } \\
\text { embryos } \\
(\%)\end{array}$ & $\begin{array}{c}\text { 16-32-cell } \\
\text { embryos } \\
(\%)\end{array}$ \\
\hline & & & \\
$18\left(37^{\circ} \mathrm{C}\right)$ & $95 / 152(63)$ & $64 / 95(67)$ & $58 / 64(90)$ \\
$3\left(33^{\circ} \mathrm{C}\right)$ & $5 / 28(18)$ & $5 / 5(100)$ & $2 / 5(40)$
\end{tabular}

Table 2. Rate of development of embryos of Monodelphis domestica in culture

$\begin{array}{lcc}\text { Number of } & \text { Stage of development } & \text { Duration of culture } \\ \text { oocytes } & \text { (cells) } & \text { (h) }\end{array}$

\begin{tabular}{lcl}
\hline 64 & 2 & $26 \pm 3$ \\
61 & 4 & $46 \pm 5$ \\
61 & $8-12$ & $59 \pm 8$ \\
58 & $16-32$ & $82 \pm 22$ \\
\hline
\end{tabular}

secretion from oviductal epithelial cells surrounds the oocyte soon after ovulation inhibiting multiple sperm penetration and polyspermy (Baggott and Moore, 1990). It would be crucial therefore that the fertilizing spermatozoon could undergo an acrosome reaction immediately on contact with the zona in vivo. In the presence of high numbers of spermatozoa and as a consequence of the lack of mucoid coat, oocytes fertilized in vitro exhibited a high rate of polyspermy when sperm-egg incubations were for more than $2 \mathrm{~h}$. In contrast, oocytes recovered from the oviduct with no discernible mucoid coat under the light microscope usually failed to be fertilized owing to the presence of a fine deposit detectable only by electron microscopy (H. D. M. Moore, unpublished observation).

Compared with the narrow penetration slit produced by the fertilizing spermatozoon of eutherian species (see Moore and Bedford, 1983), Monodelphis fertilization in vitro involved a large breach of the zona with an associated extrusion of ooplasm. The latter was probably the result of a fertilization cone forming at the site of sperm-egg fusion (Taggart et al., in press), which became exaggerated during in vitro incubation owing to the lack of mucoid coat but was later resorbed into the perivitelline space following first cleavage and the vesiculation of the ooplasm (Baggott and Moore, 1990). The majority of embryos developed to $16-32$ cells in culture, but as the unilaminar blastocyst was forming development was halted. In a previous study in our laboratory in vivo fertilized zygotes cultured in vitro stopped development mainly at the eight-cell stage 


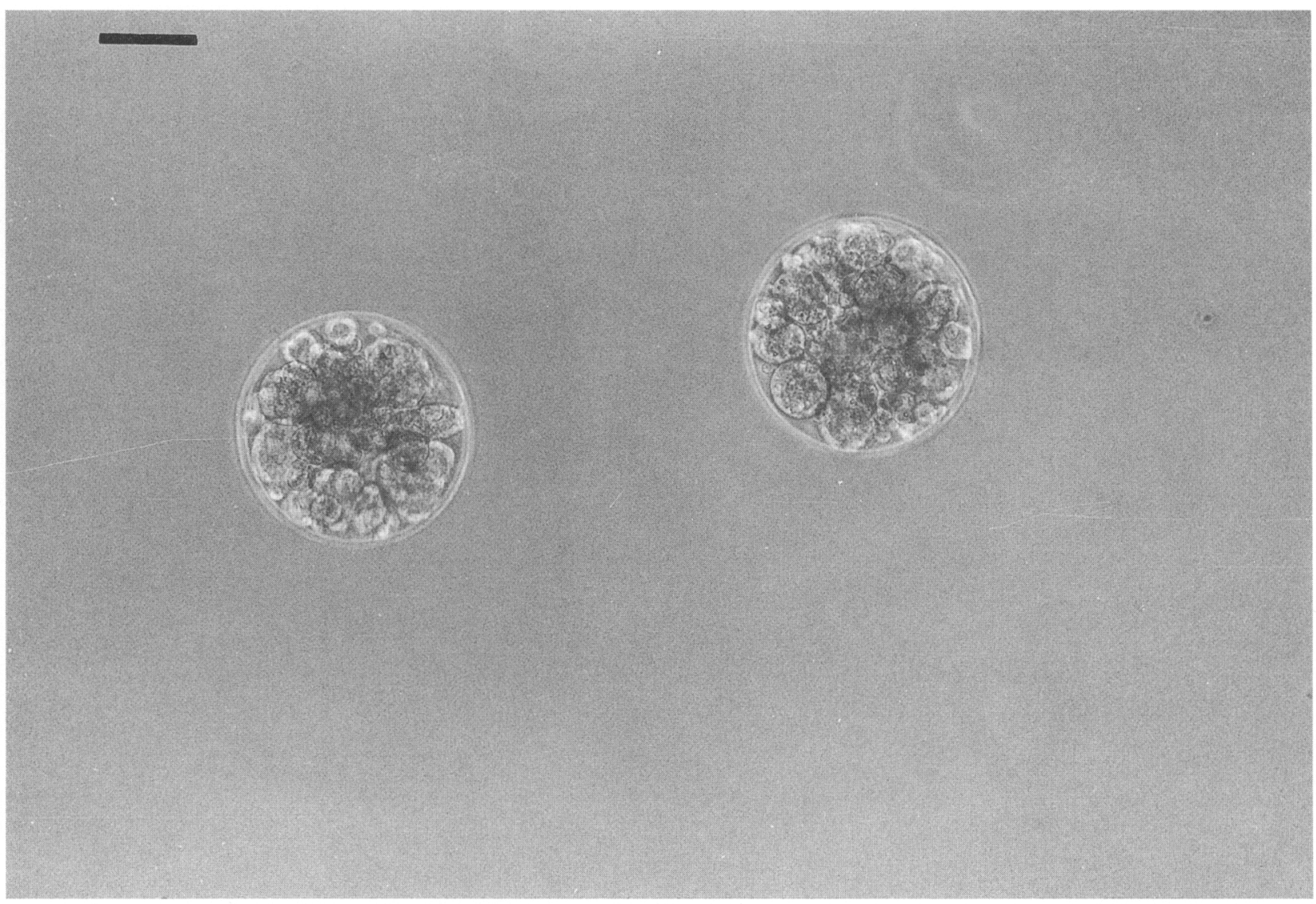

Fig. 6. A light micrograph of embryos of the grey short-tailed opossum at the 16-cell stage. Blastomeres are beginning to adhere to the inner surface of the zona pellucida before unilaminar blastocyst formation. Embryos arrested development at this stage in culture. Bar $=100 \mu \mathrm{m}$.

(Baggott and Moore, 1990). Since then the culture medium has been substantially altered and this has probably led to the improvement in the number of cell divisions. The reason for the failure at the unilaminar blastocyst stage remains unclear. Since at this time, blastomeres have to make a contiguous layer around the inner surface of the zona pellucida, particular growth factors may be necessary which were not provided in the culture medium. The semi-permeable shell membrane, normally laid down around the zygote in the uterus, may also be important for development at this time. Alternatively, the basal body temperature for Monodelphis $\left(33^{\circ} \mathrm{C}\right)$ may be a more appropriate incubation temperature. Recently, Selwood and Vandeberg (1992) demonstrated that Monodelphis embryos incubated in modified medium MEM with $10 \%$ fetal calf serum at $37^{\circ} \mathrm{C}$ completed fewer divisions, had a slightly increased rate of cleavage and showed an increased tendency to degenerate compared with embryos cultured at $32.6^{\circ} \mathrm{C}$. However, the rate of embryo development (from one cell to 16 cell: 1 of 9) at $32.6^{\circ} \mathrm{C}$ in their study was considerably lower than in the present investigation at $37^{\circ} \mathrm{C}(58$ of 64), indicating that the composition of the medium or the lack of mucoid/shell membranes (due to in vitro fertilization) may be more critical factors than the incubation temperature unless conditions are already optimal.

As far as we are aware, this is the first report of complete in vitro fertilization in a marsupial and forms the basis for the practical study of gamete and embryo development in this group of mammals. It will be important to determine whether the processes described for Monodelphis (Didelpidae) are similar in all marsupials. Moreover, several questions now deserve critical experimental analysis, including the ultrastructure of fertilization (Taggart $e t$ al., in press), the role of the fertilizing spermatozoon to repolarise the zygote and the determinitive mechanisms involved in the formation of the unilaminar blastocyst and thereafter the fetus in marsupials. Finally, it is hoped that such in vitro fertilization and embryo culture techniques can be used to help in the conservation of the many marsupials now threatened with extinction.

During this study, D. Taggart was supported by a Research Fellowship from the Journal of Reproduction and Fertility. Aspects of this work were also supported by a programme grant from the Medical Research Council and the Agricultural and Food Research Council. We thank $\mathrm{H}$. O'Brien for her technical assistance.

\section{References}

Baggott LM and Moore HDM (1990) Early embryonic development of the grey short-tailed opossum, Monodelphis domestica, in vivo and in vitro Journal of Zoology 222 623-639

Baggott LM, Davis-Butler S and Moore HDM (1987) Characterisation of oestrus and timed collection of oocytes in the grey short-tailed opossum, Monodelphis domestica Journal of Reproduction and Fertility 79 105-114 
Mate KE and Rodger JC (1991) Stability of the acrosome of the brush-tailed possum (Trichosurus vulpecula) and tammar wallaby (Macropus engenii) in vitro and after exposure to conditions and agents known to cause capacitation or acrosome reaction of eutherian spermatozoa journal of Reproduction and Fertility $9141-48$

Mollenhauer HH (1964) Plastic embedding mixtures for use in electron microscopy Stain Technology 39 111-114

Moore HDM (1992) Reproduction in the gray short-tailed opossum, Monodelphis domestica. In Reproductive Biology of South American Vertebrates, pp 229-241 Ed. WC Hamlett. Springer Verlag, New York

Moore HDM and Bedford JM (1983) The interaction of mammalian gametes in the female. In Mechanism and Control of Animal Fertilization, pp 453-497 Ed. JF Hartmann. Academic Press, New York

Rodger JC (1991) Fertilization of marsupials. In A Comparative Overview of Mammalian Fertilization, pp 117-135 Eds BS Dunbar and MG O'Rand. Plenum Press, New York

Rodger JC and Bedford JM (1982) Separation of sperm pairs and sperm-egg interaction in the opossum, Didelphis virginiana lournal of Reproduction and Fertility 64 171-179
Selwood L and Vandeberg JL (1992) The influence of incubation temperature on oocyte maturation, parthenogenetic and embryonic development in vitro in the marsupial Monodelphis domestica Animal Reproduction Science 29 99-116

Taggart DA (1991) Sperm pairing in the epididymis of the grey short-tailed opossum, Monodelphis domestica Journal of Reproduction and Fertility Abstract Series 8 Abstract, 11

Taggart DA, O'Brien HP and Moore HDM Ultrastructural characteristics of in vivo and in vitro fertilization in the grey short-tailed opossum, Monodelphis domestica American Journal of Anatomy (in press)

Temple-Smith PD and Bedford JM (1980) Sperm maturation and the formation of sperm pairs in the epididymis of the opossum, Didelphis virginiana Joumal of Experimental Zoology 214 161-171

Tyndale-Biscoe CH and Renfree MB (1987) Reproductive Physiology of Marsupials Cambridge University Press, Cambridge

Yanagimachi R (1988) Mammalian Fertilization. In The Physiology of Reproduction, pp 135-185 Eds E Knobil and J Neill. Raven Press, New York

Yanagimachi R and Chang MC (1964) In vitro fertilization of golden hamster ova Journal of Experimental Zoology 156 361-376 